ISSN 0001-6002/2002/44/3/113-116

A cta M édica Costarricense, $\bigcirc 2002$

Colegio de M édicos y Cirujanos

\title{
Colecistectomía abierta versus laparoscópica: experiencia en el Hospital San Juan de Dios
}

Juan José Zúñiga-Vargas', Julia Vargas-Carranza'

Resumen: La cirugía laparoscópica es una técnica mínimamente invasiva de uso cotidiano en el ámbito mundial. Desde hace quince años, la colecistectomía laparoscópica es un procedimiento seguro y muy valioso a disposición del cirujano.

Este estudio tiene como fin comparar los resultados de la colecistectomía laparoscópica en el Hospital San J uan de Dios con la técnica de la colecistectomía abierta ó tradicional.

M étodos: Se realizó un estudio retrospectivo de todos los pacientes colecistectomizados desde julio de 1996 a junio de 1999, en el Hospital San Juan de Dios en Costa Rica.

Al comparar las dos técnicas quirúrgicas (clásica y laparoscópica), se consideró la evolución posoperatoria y las posibles complicaciones que aparecen posteriores al acto quirúrgico. A demás se buscaron las contraindicaciones que dicha técnica pudiese tener.

Resultados : Fueron revisados un total de 3193 pacientes colecistectomizados, de los cuales 476 fueron operados por laparoscopia. Se tuvo en éstas menos complicaciones posquirúrgicas y menor estancia posoperatoria que en las colecistectomías abiertas. También se notó una disminución paulatina del tiempo quirúrgico en los actos por laparoscopia.

Conclusión: Se comprueban las ventajas de la cirugía invasiva mínima y se documenta que este tipo de abordaje es más seguro y beneficioso para el paciente y para el médico mismo.

Descriptores: colecistectomía abierta, colecistectomía laparoscópica, abordaje quirúrgico, colelitiasis, colédoco litiasis, contraindicaciones, complicaciones, tiempo quirúrgico pre y pos-operatorio, síndrome M irizzi.

Recibido: 5 de marzo del 2002

Aceptado: 30 de julio del 2002

En Costa Rica, la colecistectomía laparoscópica (CL) se introdujo a mediados de la década de los noventa siendo realizada entonces únicamente en la práctica de la medicina privada.

A mediados de 1996 se comenzó a aplicar este procedimiento como parte de la cirugía regular en el Hospital San Juan de Dios.

Describimos aquí nuestra experiencia con este abordaje durante 2 años y 11 meses.

Hospital San Juan de Dios, Caja Costarricense de Seguro Social, San José-Costa Rica.

A breviaturas: CL: Cirugía laparoscópica

Correspondencia: Julia Vargas Carranza. APDO: 19 - 2300. Correo electrónico: zuva84@ hotmail.com

\section{Materiales métodos}

El trabajo consistió en una revisión y análisis de las colecistectomías realizadas en todos los 4 servicios de Cirugía General del Hospital entre julio de 1996 a junio de 1999 que fueron anotadas en el Archivo del Hospital San Juan de Dios (el Hospital cuenta con cuatro servicios de Cirugía). Se estudiaron 3193 expedientes de los cuales 476 correspondieron a pacientes de (CL) y 2717 realizadas por el método tradicional.

Todas las CL s fueron realizadas por cirujanos generales y las colecistectomías clásicas por cirujanos general es y residentes de cirugía general.

Técnica Q uirúrgica: esta se basa en la expuesta por Reddick y OIsen ${ }^{1,2,17}$ ampliamente descrita en la literatura. 
Se estudiaron los siguientes parámetros:

a) La edad

b) El sexo

c) Las patologías asociadas en los 476 pacientes de CL

d) La elección del abordaje quirúrgico distribuido por cada servicio de cirugía general de este hospital.

e) Estancia preoperatoria y posoperatoria según el abordaje quirúrgico.

f) La necesidad de exploración de vías biliares y otras complicaciones que ocurrieron, en cada grupo.

g) Tiempo quirúrgico evaluando su comportamiento según aumentaba la experiencia del acto quirúrgico laparoscópico.

\section{Resultados}

En la cuadro 1 se muestra como paulatinamente, cada año, aumentó el número de $\mathrm{CL}$.

L os hallazgos encontrados con respecto a la distribución por edad y sexo se encuentran dentro de los mismos parámetros descritos en la literatura mundial 1. La edad, osciló entre los 11 y los 88 años, el promedio de 45 años, con una mayoría de pacientes que estaban en el grupo de 40 a 49 años, seguida por el grupo de 30 a 39 años. La distribución por sexos fue de 2637 del sexo femenino y 556 del sexo masculino. En el grupo de cirugía laparoscópica hubo 398 mujeres y 78 hombres.

\section{Técnica quirúrgica}

En los primeros casos, el pneumoperitoneo se realizaba con la aguja de Veress, pero actualmente se utiliza el trocar de Hassan. Cuando el cirujano se familiariza con este método, reconoce que es más seguro, eficiente y rápido que con la aguja de Veress. Con esto se evitaron las complicaciones descritas en la literatura como lesiones intestinales y vasculares al introducir el primer trocar a ciegas $3,4,5$.

Para evitar las lesiones de las vías biliares se siguen los nueve pasos recomendados por Asbun y Rossi de la Clínica Lahey ${ }^{6,7}$.

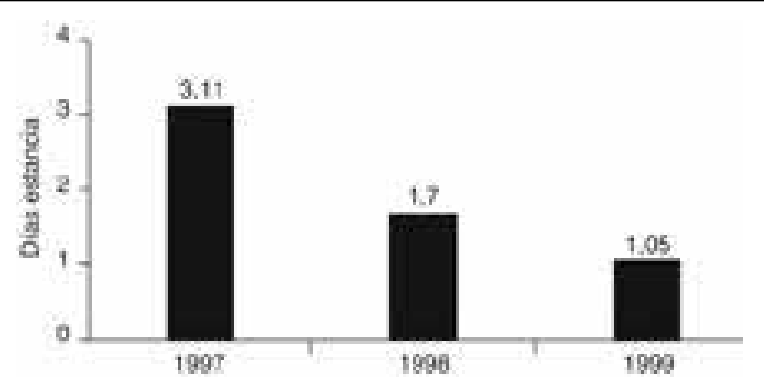

Figura 1: Estancia posoperatoria pacientes colecistectomisados Técnica laparoscópica HSJD.
Patologías asociadas

Se real izaron dos colecistectomías laparoscópica en pacientes embarazadas de 21 y 18 semanas de embarazo, sin que se presentare ninguna dificultad técnica, ni complicación transoperatoria (cuadro 2). Se introdujo una presión intraabdominal por debajo de los $10 \mathrm{mmHg}$ y ninguna de las dos pacientes presentó contracciones uterinas posoperatorias.

\section{Estancia posoperatoria}

El $65 \%$ de los pacientes de CL se egresaron dentro de las siguientes 24 horas, mientras que sólo el $15 \%$ de los pacientes operados por la técnica abierta, fueron egresados durante ese período. Esto se puede ver en la figura 1.

A las 48 horas, se egresaron más del $85 \%$ de los pacientes sometidos a $\mathrm{CL}$ comparado con el $58 \%$ de los pacientes operados por la técnica clásica. El promedio de estancia para este último grupo fue de 4 días.

En 1997 el promedio de estancia fue de 3,11 días, en 1998 bajó a 1.7 días y en 1999 disminuyó hasta 1.05 días.

Abordaje quirúrgico

En promedio el $15 \%$ de todas las colecistectomías fueron realizadas por laparoscopía. Durante el semestre analizado de 1996 la laparoscopías representaron sólo el 0,98\% de todas las colecistectomías, al año siguiente fueron el 4,54\%, en 1998 y en el primer semestre de 1999 habían subido ya al 27\%.

\begin{tabular}{|lllll|}
\hline \multicolumn{5}{|c|}{ Cuadro 1 } \\
Distribución anual de las colecistectomías \\
\hline AÑO & ABIERTA & LAPAROSCOPICA & TOTAL & $\%$ de \\
& & & & laparoscópica \\
\hline 1996 & 510 & 5 & 515 & 0.98 \\
1997 & 1056 & 48 & 1104 & 4.54 \\
1998 & 675 & 249 & 920 & 27 \\
1999 & 490 & 174 & 654 & 26.6 \\
\hline TOTAL & 2717 & 476 & 3193 & 14.9 \\
\hline
\end{tabular}

\begin{tabular}{|lc|}
\hline \multicolumn{2}{|c|}{ Cuadro 2 } \\
Patologías médicas asociadas \\
\hline Patologías asociadas & Número de casos \\
\hline Hipertensión arterial & 48 \\
Obesidad & 18 \\
Colecistitis & 17 \\
Diabetes Mellitus & 12 \\
Pancreatitis & 10 \\
Adherencias & 10 \\
Cardiopatías & 8 \\
Neumopatías & 7 \\
Cirrosis & 2 \\
Vesícula en porcelana & 2 \\
Piocolecisto & 2 \\
Quiste renal & 1 \\
Aneurisma cerebral & 1 \\
Drepanocitosis & 1 \\
\hline
\end{tabular}


Tomando en cuenta todos los servicios de Cirugía General el $64 \%$ de los casos se realizaron en Cirugía 1,el 10\% en Cirugía 2, el $11.5 \%$ en Cirugía 3 y el $14.5 \%$ Cirugía 4.

Exploración de vías biliares

Durante la $C L$ dos pacientes se convirtieron a cirugía abierta y se les realizó exploración del colédoco; una de ellas tenía un síndrome de M irizzi, y la segunda paciente se convirtió por presentar adherencias encontrándose además colédocolitiasis a la pal pación.

La incidencia de este hallazgo en la técnica abierta fue de $5.9 \%$ (189 pacientes).

En 3 casos se real izó pancreatografía retrógrada endoscópica con papilotomía y extracción de cálculos y luego $\mathrm{CL}$ en las siguientes 24 horas con buena evolución.

\section{Conversión}

De las 476 cirugías, en 12 casos se recurrió a la conversión de $C L$ a colecistectomía abierta o clásica, esto corresponde al $2.51 \%$. En la literatura mundial se reportan porcentajes que oscilan entre $0.08 \%$ y $14 \% 8.9$.

Las conversiones de CL a cirugía clásica ocurrieron por diferentes razones: 2 por dificultad con el insuflador, 2 por sangrado de la arteria cística, 2 por escape de bilis debido al desplazamiento de las grapas del conducto cístico, 1 por el hallazgo anatómico anómalo de un conducto accesorio que drenaba en el cístico, 1 paciente con síndrome de M irizzi III, 2 pacientes con adherencias; uno de estos casos operado anteriormente de cáncer gástrico y por último, 1 por lesión del colédoco durante el acto quirúrgico. Este fue reparado inmediatamente y no tuvo complicaciones posoperatorias.

U na paciente femenina de 44 años desarrolló estenosis severa del conducto hepático común que ameritó una anastomosis hepatoyeyunal cuatro meses después, actualmente (24 meses después) está asintomática.

L esión de la vía biliar

Solo en dos mujeres ocurrió esta complicación. Una de ellas fue reconocida prontamente y fue reparada sin complicaciones, la otra paciente es la descrita en el párrafo anterior.

Esto representa el $0,42 \%$ contra el $1,04 \%$ de los operados con la técnica clásica.

Inicialmente, se dijo de esta nueva técnica que producía lesiones de las vías biliares con mayor frecuencia. Sin embargo los últimos reportes muestran un cambio en relación a las colecistectomías abiertas ${ }^{10,11,12,13,14,15}$.

Tiempo Quirúrgico

Comparando los tiempos quirúrgicos de ambas técnicas observamos que el $9.25 \%$ de los pacientes fueron intervenidos con la técnica abierta en menos de 30 minutos, en tanto que sólo el $2,4 \%$ de los intervenidos por laparoscopia lo fueron en ese tiempo.
El $85 \%$ de los pacientes operados por la técnica abierta, lo son en menos de 90 minutos, en contraposición con el $76 \%$ de los operados por laparoscopia.

A pesar de lo anterior es importante analizar la evolución del tiempo quirúrgico de las $C L$ con el avance de los años: en 1997 se duró un promedio de 88.12 minutos; en 1998 éste disminuyó a 73.27 minutos y en 1999 bajó hasta 68.7 minutos.

El promedio general para las colecistectomías abiertas fue de 63.34 minutos.

\section{Complicaciones}

De los 476 pacientes operados por CL, 14 tuvieron complicaciones, lo que representa el $2.94 \%$ de los mismos.

Las complicaciones más frecuentes fueron:

1) Sangrado del lecho vesícular, esta situación, la más frecuente, ocurrió en cuatro ocasiones.

2) Dos pacientes cursaron con dolor posquirúrgico y su estancia hospitalaria se prolongó por 48 horas.

3) Dos pacientes, a quienes se les encontró piocolecisto durante la cirugía laparoscópica, desarrollaron abscesos subhepáticos, que no necesitaron reintervención

4) Dos pacientes desarrollaron infección de la herida umbilical. Uno de ellos posteriormente desarrolló una hernia en ese sitio. Un paciente presentó íleo paralítico.

5) Un paciente de 75 años murió de insuficiencia cardiaca refractaria posterior a la reposición de líquidos indicados para tratar hipotensión e hipovolemia por sangrado del lecho vesicular, convirtiéndose en la única muerte en este reporte $(0.21 \%$ de mortalidad).

Solamente un $11 \%$ de los pacientes de $C L$ requirió 3 o más días de hospitalización mientras que con la técnica abierta el porcentaje fue mayor del $40 \%$.

\section{Discusión}

La primer colecistectomía laparoscópica que se realizó en el mundo fue en 1986, en A lemania por Eric M ühe. Dos años después, este procedimiento se popularizó al resto del orbe ${ }^{16}$. En el campo de la Cirugía General desde 1996 en nuestro país, se realizan cada vez más intervenciones laparoscopicas, hoy se hacen no solo colecistectomías sino que podemos citar el tratamiento quirúrgico de la enfermedad ácido péptica, de las hernias hiatales y de las hernias inguinales.

$\mathrm{La} C L$ a pesar de ser una técnica relativamente nueva ha demostrado ser un procedimiento seguro y técnicamente sencillo. Es importante recordar que en la técnica laparoscópica se hacen tres incisiones: umbilical, epigástrica y una en la línea axilar media derecha, $2 \mathrm{~cm}$ por abajo del reborde costal, ninguna de las tres es mayor de $1 \mathrm{~cm}$ de longitud en comparación con la herida que se realiza en la 
colecistectomía clásica que nunca es menor de $10 \mathrm{~cm}$. Solo dos pacientes intervenidos por laparoscopía presentaron dehiscencia o infección de la herida quirúrgica ${ }^{1,17}$.

En el Hospital San Juan de Dios, solamente un 27\% de las colecistectomías se realizan por laparoscopía, a pesar que de que esta técnica en este mismo trabajo ha demostrado enormes ventajas en comparación con la técnica convencional, como lo son:

1) Una baja tasa de complicaciones, aproximadamente tres veces menor que en las colecistectomías abiertas. Dato que es comparable con lo que se describe la literatura mundial.

2) Una estancia hospitalaria posoperatoria menor y una recuperación más pronta del paciente, consecuentemente menos incapacidad laboral.

3) M enor dolor posoperatorio.

4) M ejores resultados estéticos al tener menor tamaño la herida quirúrgica y mejor cicatrización por esta misma causa.

No hemos encontrado contraindicaciones para realizar la cirugía por este abordaje. Pacientes neumópatas, cardiópatas, cirróticos, embarazadas, colecistitis agudas y piocolecistos, se han beneficiado sin que aumenten las complicaciones, como se puede ver en la cuadro 2 .

Cuando los pacientes presentan colédoco litiasis, hemos tenido magníficos resultados realizando primero extracción endoscópica de los cálculos y posteriormente la C.L, pero esto ha sido en un número reducido de pacientes, esperamos contar a corto plazo con más pacientes para tratarlos de esta manera.

Finalmente, es indiscutible el beneficio que significa para el Hospital y para la Caja Costarricense del Seguro Social, la reducción de la estancia posoperatoria, la menor cantidad de complicaciones y la satisfacción del asegurado.

\section{Abstract}

J ustification: Today, laparoscopic surgery as a least invasive technique is widely used in the world. Laparoscopic cholecystectomy is a valuable and safe technique and is has been available to the general surgeon for the last 15 years.

In Costa Rica it has become a pretty common procedure with great results but they have not been well studied and documented.

This study aims to expose part of the work that takes place at the Hospital San Juan de Dios with laparoscopic cholecystectomy and highlights the results in contrast with those of traditional cholecystectomy.
M ethodology: A retrospective study was conducted of all the cholecystectomies performed at the Hospital San Juan de Dios in Costa Rica from July 1996 to J une 1999

A comparative analysis between the classic and the laparoscopic approach was completed considering postoperative evolution and postoperative complications.

Results: A total of 3193 cholecystectomies, were reviewed, 476 were laparoscopic cholecystectomies, they had less postoperative complications and less post-operative recovery time as opposed to the open cholecystectomies. The study showed further a progressive diminution of the operative time in the laparoscopic group.

Conclusions: Laparoscopic cholecystectomy is a safe procedure and it carries several advantages both for the patient and the surgeon.

\section{Referencias}

1. A sbun H, Rossi R. Techniques of L aparoscopic Cholecystectomy: The Difficult O peration, Surg. Clin. N. A, 1994; 74:755-775.

2. Lujan J., Parrilla P., Robles R., M arin P., Torralba J., Garcia-A yllon J; Laparoscopic Cholecystectomy vs. Open Cholecystectomy in the treatment of A cute Cholecystitis, A rch. Surg, 1998;133: 173-175.

3. Horvath K. Strategies for the Prevention of L aparoscopic Common Bile Duct Injuries, Surg. Endosc, 1993; 7: 439-444.

4. Deziel D. Complications of Cholecystectomy. Surg. Clin. N. A ., 1994; 74:809-823.

5. Baev S., Pzarliev, Todorov G., Laparoscopic Cholecystectomy: 700 Consecutive Cases. Int. Surg, 1995; 80: 296-298.

6. Dadidoff A., Pappas T. M echanisms of Mayor Biliary Injury during Laparoscopic Cholecystectomy. A nn. of Surg, 1992; 215: 196-202.

7. Asbun H, Rossi R, Bile Duct Injury during Laparoscopic Cholecystectomy, M echanism of Injury, Prevention and $M$ anagement. World J. Surg, 1993;17:547-552.

8. Kuster G., Domagk D. Laparoscopic Cholecystectomy with delayed Cholecystectomy as an alternative to convert to open procedure. Surg. Endosc, 1996; 10: 426-428.

9. Martin R., Rossi R.. Spectrum, Mechanisms of Injury and their Prevention. Surg.Clin.N.A, 1994; 74:781-807.

10. Branum G., Schamitt C., Baillie j. Management of Major Biliary Complications after Laparoscopic Cholecystectomy, Ann. of Surg, 1993; 217: 532-541.

11. Gerhard R., Jatzko, Lisborg P., Perti A., Stettner H. Multivariate Comparison of Complications after Laparoscopic Cholecystectomy and Open Cholecystectomy, A nn. of Surg, 1995; 211: 381-386.

12. Horvath $\mathrm{K}$ Strategies for the Prevention of $\mathrm{L}$ aparoscopic Common Bile Duct Injuries. Surg. Endosc, 1993; 7: 439-444.

13. Morgenstern L., Berci G., Pasternak E. Bile Leakage after Cholecystectomy: a Laparoscopic Perspective. Surg. Endosc, 1993; 7: 432-438.

14. Rossi R. Laparoscopic Bile Duct Injuries. Risk Factors, Recognition and repair. A rch. Surg, 1992; 127: 596-602.

15. Sandberg A ., A liender G. A ccidental Lesion of the common Bile Duct at Cholecystectomy: Pre and Perioperative Factor of Importance. A nn. Surg, 1985; 201: 328-332.

16. Cuschieri A.. Cirugía Biliar Laparoscópica, Blackwell Scientific Publications. Segunda edición, España, 1994; pag. 01-101.

17. Cuschieri A., The European Experience with Laparoscopic Cholecystectomy. A m. J. Surg., 161: 385-387, 1991. 